Kong. Res. J. 2(1) : 50-52, 2015

ISSN 2349-2694

Kongunadu Arts and Science College, Coimbatore.

\title{
A STUDY ON FUZZY SHORTEST ROUTE ALGORITHM FOR TELEPHONE LINE CONNECTION
}

\author{
Vijayalakshmi, D. and R. Kalaivani* \\ Department of Mathematics, Kongunadu Arts \& Science College, Coimbatore-641 029, India \\ *E-mail: kalaivanirm@yahoo.com
}

\begin{abstract}
In computer science, there are many algorithms that finds a minimum spanning tree for a connected weighted undirected fuzzy graph. The minimum length (or cost) spanning tree problem is one of the nicest and simplest problems in network optimization, and it has a wide variety of applications. The problem is to find a minimum cost (or length) spanning tree in G. Applications include the design of various types of distribution networks in which the nodes represent cities, centers etc.; and edges represent communication links (fiber glass phone lines, data transmission lines, cable TV lines, etc.), high voltage power transmission lines, natural gas or crude oil pipelines, water pipelines, highways, etc. The objective is to design a network that connects all the nodes using the minimum length of cable or pipe or other resource.In this paper we find the solution to the problem is to minimize the amount of new telephone line connection using matrix algorithm with fuzzy graph.
\end{abstract}

Keywords: Spanning tree, minimum spanning tree, fuzzy graph, Matrix algorithm.

\section{INTRODUCTION}

A minimum cost of the fuzzy spanning tree is spanning tree, but it has weight or length associated with the edges and total weight of the tree is minimum. A fuzzy tree for that graph would be a subset of those paths that has no cycles but still connects to every vertex. There might be several spanning trees possible. A minimum fuzzy tree would be one with the lowest total cost. A less obvious application is that the minimum fuzzy spanning tree can be used to approximately solve the traveling salesman problem (Hassan, 2012).

In this paper, we find the solution for the problem, (Abhilasha, 2013) that (A.V.V.M. Sri Pushpam College, (Autonomous), Poondi, Thanjavur District, South India) needs to connect updated intercom lines connecting all the departments using a fuzzy shortest route algorithm (Amit Kumar and Manjot Kaur, 2011). The problem is to minimize the amount of new line using matrix Algorithm with fuzzy graph.

\section{Definition}

\subsection{Definition}

A fuzzy graph with $V$ as the underlying set is a pair G: $(A, \Gamma)$ where $A: V--0,0,1]$ is a fuzzy subset, $\Gamma$ : $V x V$------> $[0,1]$ is a fuzzy relation on the fuzzy subset $A$, such that $\Gamma(\mathrm{u}, \mathrm{v}) \leq \mathrm{A}(\mathrm{u}) \cap \mathrm{A}(\mathrm{v})$ for all $\mathrm{u}, \mathrm{v} \in \mathrm{V}$ (Antony Xavior et al., 2013).

\subsection{Definition}

A fuzzy Hamiltonian circuit is a circuit that visits every vertex in a fuzzy graph once with no repeats, being a fuzzy Hamiltonian circuits must start and end at the same vertex.

\subsection{Definition}

A fuzzy Hamiltonian path is a path that passes through each of the vertices in a fuzzy graph exactly once.

\subsection{Definition}

A fuzzy spanning tree is a fuzzy tree which covers all the vertices of a fuzzy graph.

NOTE 2.1: Fuzzy tress has no circuits, and it is fine to have vertices

\subsection{Definition}

A fuzzy graph $\hat{G}=(V, \sigma, \mu)$ is called a complete fuzzy graph (Nivethana and Parvathi, 2013)

if $\mu(u v)=\sigma(u)^{\bigcap} \sigma(v)$ for all $u, v \in V$ and $u v \in E$. We denote this complete fuzzy graph by Ĝk.

\section{MATRIX ALGORITHM}

Let $\mathrm{G}=(\mathrm{V}, \mathrm{E})$ be an undirected connected weighted graph with $n$ vertices, where $\mathrm{V}$ is the set of vertices, $\mathrm{E}$ is the set of edges and $\mathrm{W}$ be the set of weights (cost) associated to respective edges of the graph. Where

$\mathrm{e}_{\mathrm{ij}}$, the edge adjacent to vertices $\mathrm{v}_{i}$ and $\mathrm{v}_{j}$. 
$\mathrm{W}_{\mathrm{ij}}$, the weight associated to the edge $\mathrm{e}_{\mathrm{ij}}$.

The Weight Matrix $M$ of the graph $G$ is constructed as follows:

If there is an edge between the vertices $v_{i}$ to $v_{j}$ in $\mathrm{G}$ then Set,

$$
\mathrm{M}_{[\mathrm{i}, \mathrm{j}]}=\mathrm{w}_{\mathrm{ij}}
$$

Else

Set, $M_{[i, j]}=0$

Input: the weight matrix $\mathrm{M}=\left[\mathrm{w}_{i j}\right]_{\mathrm{n} \times \mathrm{n}}$ for the undirected weighted graph $\mathrm{G}$

\section{Step 1: Start}

Step 2: Repeat Step 3 to Step 4 until all (n-1) elements matrix of $M$ are marked or set to zero or in other words all the nonzero elements are marked

Step 3: Search the weight matrix M by rowwise to find the unmarked nonzero minimum element $M[i, j]$, which is the weight of the corresponding edge $\mathrm{e}_{\mathrm{ij}}$ in $\mathrm{M}$.

Step 4: If the corresponding edge $\mathrm{e}_{\mathrm{ij}}$ of selected $\mathrm{M}_{[\mathrm{i}, \mathrm{j}]}$ forms cycle with the already marked elements in the elements of the $M$ then Set $M_{[i, j]}=0$ else mark $\mathrm{M}_{[\mathrm{i}, \mathrm{j}]}$

Step 5: Construct the graph $\mathrm{T}$ including only the marked elements from the weight matrix $M$ which shall be the desired Minimum cost spanning tree of G.

Output: Minimum Cost Spanning Tree T of G.

\section{Example:}

In this example we applied the matrix algorithm to plan and connect an efficient fuzzy route telephone line connection for the problem (Nirmala and Uma, 2012). Consider each department as vertex such as $\mathrm{x}_{1}$-office room, $\mathrm{x}_{2}$ - mathematics, $\mathrm{x}_{3}-$ economics, $\mathrm{x}_{4}-$ History, $\mathrm{x}_{5}-$ Computer Science, $\mathrm{x}_{6}-$ Library, $\mathrm{x}_{7}-$ Physics, $\mathrm{x}_{8}-$ Chemistry, $\mathrm{x}_{9}-$ Botany and $\mathrm{x}_{10}$ - Physical education. The distance between them are represented as fuzzy weights matrix shown in the fig.3.8.

In this section, we find the solution for the below matrix the table is taken from (Nirmala and Uma, 2012) needs to connect updated intercom lines connecting all the departments exactly once. The problem is to minimize the amount of new line using Matrix Algorithm (Vijayalakshmi and Kalaivani, 2014) with fuzzy graph. The step by step procedure is given below.

Distance between the departments is shown in below matrix:

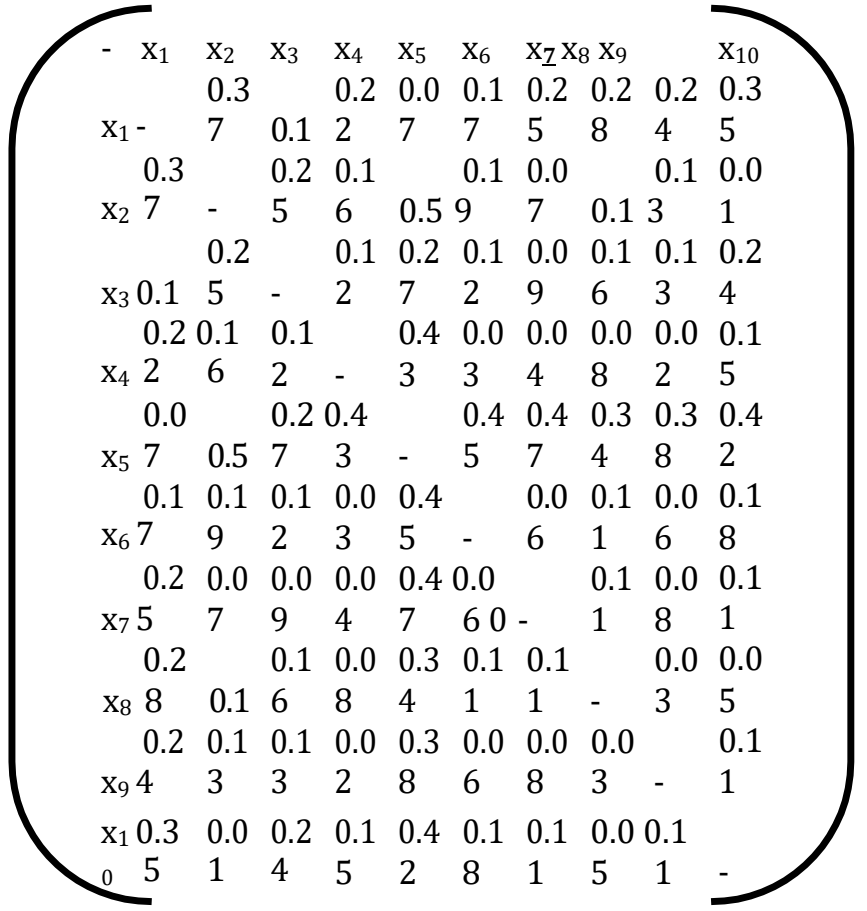

Fig. 2.2: Weight matrix.

From the above fig 3.7 the minimum weight is selected and colored, their corresponding edges were drawn repeat the process until the algorithm terminates. While drawing the graph if its forms fuzzy circuit, we remove that and in the table the weight is marked as 0 .

\begin{tabular}{|c|c|c|c|}
\hline \multirow{2}{*}{\multicolumn{2}{|c|}{$\mathrm{x}_{2}$ to }} & \multirow[b]{2}{*}{$\mathrm{x}_{10}$} & 0.01 \\
\hline & & & \\
\hline $\mathrm{x}_{4}$ & to & $\mathrm{X}_{9}$ & 0.02 \\
\hline $\mathrm{x}_{4}$ & to & $\mathrm{x}_{6}$ & 0.03 \\
\hline $\mathrm{x}_{4}$ & to & $\mathrm{X}_{7}$ & 0.04 \\
\hline $\mathrm{X}_{8}$ & to & $\mathrm{x}_{10}$ & 0.05 \\
\hline $\mathbf{X}_{7}$ & to & $\mathbf{x}_{6}$ & $\begin{array}{l}\text { ( forms fuzzy circuit) its shown in } \\
\text { belowFig.3.7) }\end{array}$ \\
\hline $\mathrm{x}_{1}$ & to & $\mathrm{X}_{5}$ & 0.07 \\
\hline $\mathrm{x}_{4}$ & to & $\mathrm{X}_{8}$ & 0.08 \\
\hline $\mathrm{x}_{3}$ & to & $\mathrm{x}_{7}$ & 0.09 \\
\hline $\mathrm{x}_{1}$ & to & $\mathrm{X}_{3}$ & 0.1 \\
\hline
\end{tabular}

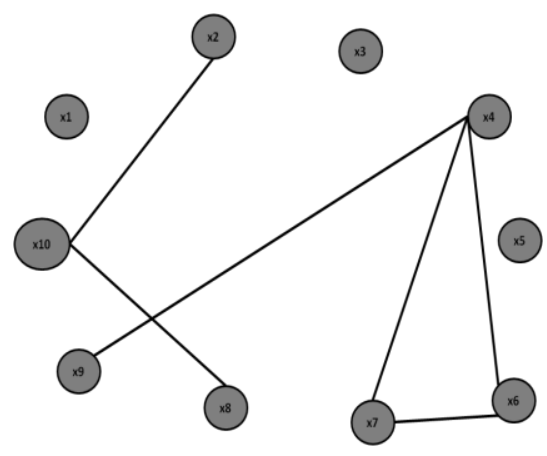

Fig. 2.3: Fuzzy circuit $\left\{\mathrm{x}_{4}, \mathrm{x}_{6}, \mathrm{x}_{7}\right\}$ 


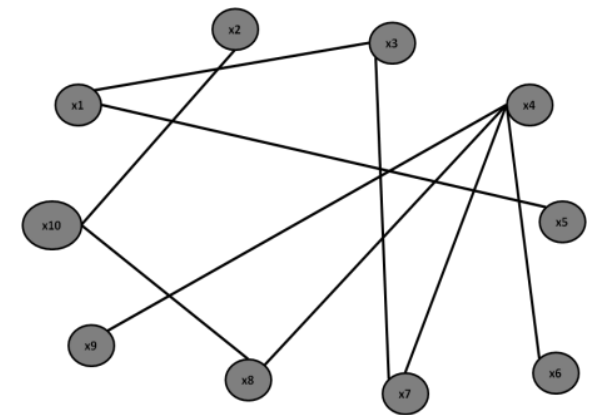

Fig 2.4: Updated fuzzy route telephone line connection

Using the Matrix Algorithm

The length of the cable

$=\left\{\left(\mathrm{x}_{2}, \mathrm{x}_{10}\right)+\left(\mathrm{x}_{4}, \mathrm{X}_{9}\right)+\left(\mathrm{x}_{4}, \mathrm{X}_{6}\right)+\left(\mathrm{x}_{4}, \mathrm{X}_{7}\right)+\left(\mathrm{x}_{8}, \mathrm{X}_{10}\right)+\left(\mathrm{x}_{1}, \mathrm{x}_{5}\right)+\left(\mathrm{x}_{1}\right.\right.$, $\left.\left.\mathrm{x}_{3}\right)+\left(\mathrm{x}_{4}, \mathrm{x}_{8}\right)+\left(\mathrm{x}_{3}, \mathrm{x}_{7}\right)\right\}$

$=$

$0.01+0.02+0.03+0.04+0.05+0.07+0.08+0.09+0.1$

$$
=0.49 \text {. }
$$

\section{CONCLUSION}

In this paper we apply the Matrix Algorithm for the problem that connecting distances to plan and visit an efficient fuzzy spanning tree route for the intercom land line (phone) to reach it all the department exactly once. So we conclude that Matrix Algorithm is the best to adopt for these types of problems.

\section{REFERENCES}

Abhilasha, R. ( 2013). Minimum cost spanning tree using prim's Algorithm. Int. J. Adv. Res. Comp. Sci. Manag. Stru. 1(1).

Amit Kumar and Manjot Kaur (2011). A new algorithm for solving network flow problems with fuzzy arc lengths. Turk. J. Fuzzy Sys. 2(1).

Antony Xavior, D., D. Florence Isido and V.M. Chtitra (2013). On Domination in fuzzy graphs. Int. J. Comp. Alg. 2: 248-250.

Hassan (2012). An efficient method to solve least cost minimum spanning tree. Comp. Inform. Sci. 24: 101-105.

Nirmala, G. and K. Uma (2012). Fuzzy Shortest Route Algorithm for Telephone Line Connection, Int. J. Sci. Res. Pub. 2.

Nivethana, V. and A. Parvathi (2013). Fuzzy Total Coloring and Chromatic Number of a Complete Fuzzy Graph. Int. J. Emerg. Trends Eng. Dev. 6(3).

Vijayalakshmi, D., R. Kalaivani (2014). Minimum Cost Spanning tree using Matrix Algorithm. Int. J. Sci. Res. Pub. 4(9). 